\title{
On elliptic curves with a closed component passing through a hexagon
}

\author{
Miroslav Kureš
}

\begin{abstract}
In general, there exists an ellipse passing through the vertices of a convex pentagon, but any ellipse passing through the vertices of a convex hexagon does not have to exist. Thus, attention is turned to algebraic curves of the third degree, namely to the closed component of certain elliptic curves. This closed curve will be called the spekboom curve. Results of numerical experiments and some hypotheses regarding hexagons of special shape connected with the existence of this curve passing through the vertices are presented and suggested. Some properties of the spekboom curve are described, too.
\end{abstract}

\section{Introduction}

In many practical applications, we need to find a curve passing through given points. There are also known procedures for a construction of a curve, for example, from its raster image: see the situation for the ellipse described by J. Hrdina and J. Pavlík in [3]. So-called Hough transform was introduced in image processing for the automatic detection of straight lines in images. The idea behind this computational tool is that, in a typical parametric representation, a straight line is uniquely represented by a pair of parameters: the slope and the intercept. Therefore a point on a straight line in the image space is projected into a straight line in the parameter space, and the whole

Key Words: Algebraic closed curves, elliptic curve, hexagon.

2010 Mathematics Subject Classification: Primary 14H52, 65D10.

Received: April, 2018.

Revised: July, 2018.

Accepted: July, 2018. 
straight line in the image space is projected into a single point in the parameter space: this is the intersection point of all projected straight lines. In the paper [2] of M. C. Beltrametti, A. M. Massone and M. Piana the generalized method is used for the automated recognition of rational cubic, quartic, and elliptic curves. The accuracy of this approach is tested against synthetic data and in the case of experimental observations provided by the NASA Solar Dynamics Observatory mission. It is interesting that elliptic curves are recognized in images of the solar corona and cromosphere in the ultraviolet and extreme ultraviolet wavelengths (on data provided by Solar Dynamics Observatory $\mathrm{SDO}$ ), see [2]. An interesting occurrence of elliptic curves in the natural sciences is also described in G. S. Kopp's paper [4], which states that linear wave solutions to the Charney-Hasegawa-Mima partial differential equation with periodic boundary conditions have two physical interpretations: Rossby (atmospheric) waves, and drift (plasma) waves in a tokamak. These waves display resonance in triads. Primitive resonant triads are identified as rational points on a rational elliptic surface having elliptic curves as fibers.

In our research, we will deal with simple closed curves, and we will not leave elliptic curves at the same time. How do we get to this point of view?

When viewing simple closed curves, let us restrict ourselves to algebraic curves. It is well known that there is a suitable second degree curve, an ellipse with the equation

$$
k(x-a)^{2}+l(y-b)^{2}=1,
$$

where $a, b, k, l \in \mathbb{R}, k>0, l>0\left([a, b]\right.$ is the center of the ellipse and $\frac{1}{\sqrt{k}}, \frac{1}{\sqrt{l}}$ its semi-axises.).

It is not difficult to generalize this notorious example. An elementary way can be, for example, by a taking of the fourth degree curve

$$
k(x-a)^{4}+l(y-b)^{4}=1,
$$

where $a, b, k, l \in \mathbb{R}, k>0, l>0$, or, more generally, every curve of an even order of the form *

$$
k(x-a)^{2 n}+l(y-b)^{2 n}=1,
$$

$a, b, k, l \in \mathbb{R}, k>0, l>0, n \in \mathbb{N}$.

\footnotetext{
*i.e. even order superellipses first discussed by Gabriel Lamé in 1818
} 

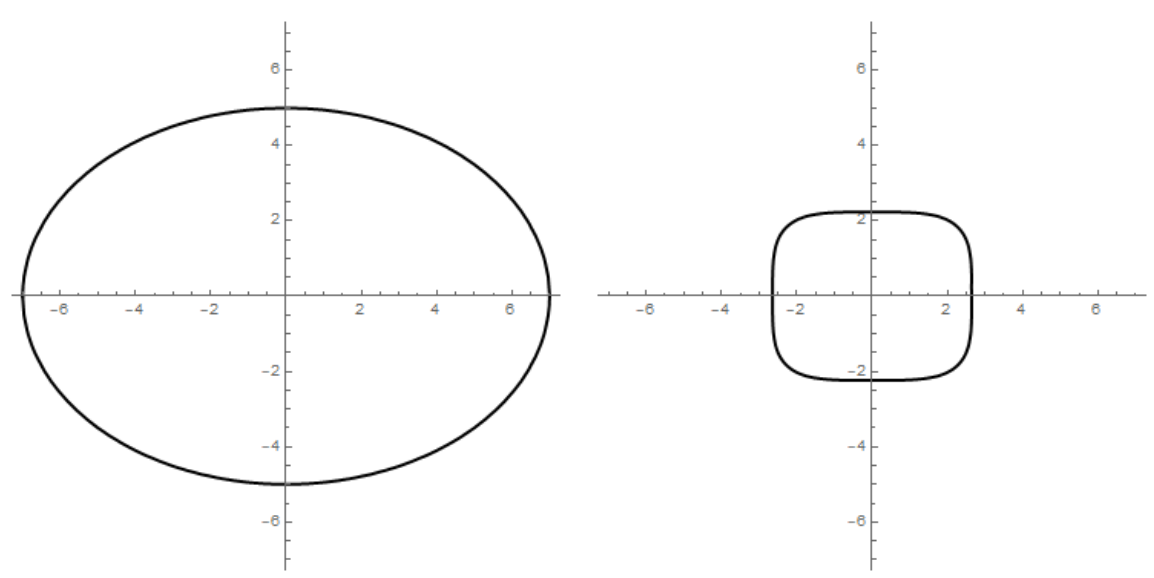

Figure 1. Closed curves $k(x-a)^{2}+l(y-b)^{2}=1$ and $k(x-a)^{4}+l(y-b)^{4}=1$, where $a=b=0, k=\frac{1}{49}, l=\frac{1}{25}$.

A more refined and sophisticated method is the perturbation of the curves. The idea is to take the union of two curves, and then slightly modify this union to get an irreducible smooth curve. Let us take for example the ellipse

$$
\frac{1}{49} x^{2}+\frac{1}{25} l(y-b)^{2}-1=0
$$

and the line

$$
y-5=0
$$

We obtain the equation

$$
\left(\frac{1}{49} x^{2}+\frac{1}{25} l(y-b)^{2}-1\right)(y-5)=0
$$

which is a cubic curve. Now, we take instead the equation

$$
\left(\frac{1}{49} x^{2}+\frac{1}{25} l(y-b)^{2}-1\right)(y-5)=t
$$

with small positive $t$. See the figure below, where we have a "new" cubic curve with two components, where one compoment is simple and closed. 

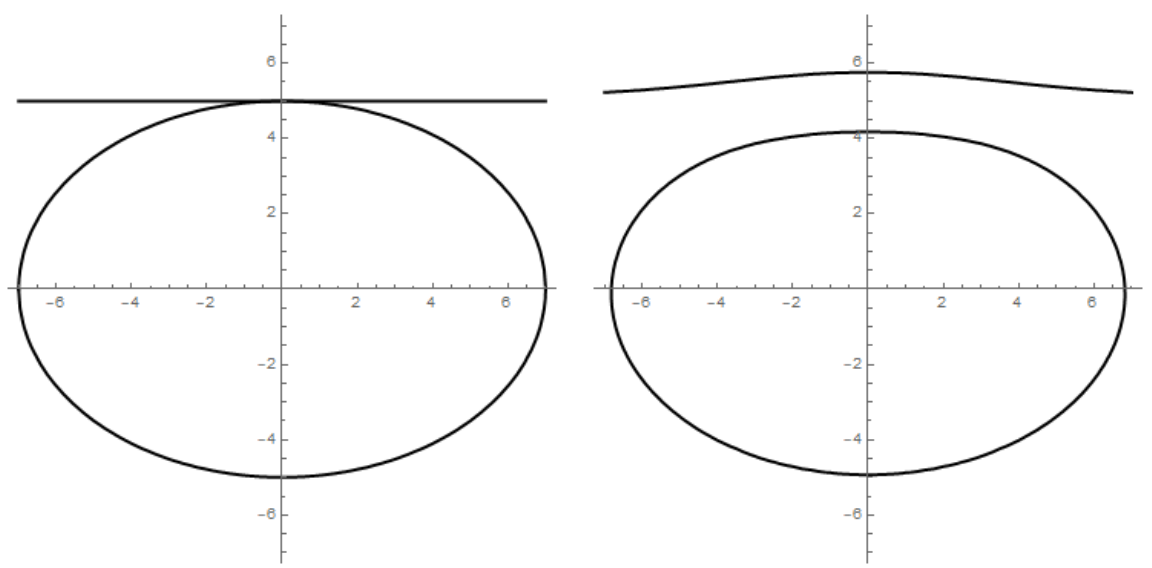

Figure 2. Cubic curves $\left(\frac{1}{49} x^{2}+\frac{1}{25} y^{2}-1\right)(y-5)=0$ and $\left(\frac{1}{49} x^{2}+\frac{1}{25} y^{2}-1\right)(y-5)=\frac{1}{4}$.

In particular, if we focus on ellipses, we recall the fundamental result of M. Agarwal, J. Clifford and M. Lachance.

Theorem 1. ([1]) Inscribed ellipses in convex non-degenerate $n$-gons:

(i) In triangles there exists a unique two-parameter family of inscribed ellipses.

(ii) In quadrilaterals there exists a unique one-parameter family of inscribed ellipses.

(iii) In pentagons there exists precisely one inscribed ellipse.

(iv) For $n \geq 6$, there exist $n$-gons for which there are no inscribed ellipses; whenever there is an inscribed ellipse, it is unique.

Next, we will deal with other cubic curves, namely elliptic curves. However, we will not get the result of the quality of the previous theorem, but our work will highlight some phenomena important for further research.

\section{The elliptic curve and the spekboom curve}

\subsection{The elliptic curve we are considering}

We start with the elliptic curve of a form

$$
(y-d)^{2}=k(x-a)(x-b)(x-c)
$$


where $a, b, c, d \in \mathbb{R}, k \in \mathbb{R}^{+}, a, b, c$ mutually different, so without loss of generality, we may assume $a<b<c$.

We use the linear transformation

$$
\begin{aligned}
x & =\frac{1}{\sqrt[3]{k}} \bar{x}+\frac{1}{3}(a+b+c) \\
y & =\bar{y}+d
\end{aligned}
$$

and obtain the usual equation of the elliptic curve

$$
\bar{y}^{2}=\bar{x}^{3}+p \bar{x}+q
$$

where

$$
\begin{aligned}
p & =\frac{\sqrt[3]{k^{2}}}{3}\left(a b+a c+b c-a^{2}-b^{2}-c^{2}\right) \\
q & =\frac{k}{27}(a+b-2 c)(a+c-2 b)(b+c-2 a) .
\end{aligned}
$$

The discriminant is

$$
-16\left(4 p^{3}+27 q^{2}\right)=16 k^{2}(a-b)^{2}(a-c)^{2}(b-c)^{2}
$$

what is evidently positive just as it should be for a non-singular elliptic curve having two components. One component is unbounded and the second one is bounded and closed.

To get a completely general form, we apply a rotation

$$
\begin{aligned}
& x=\bar{x} \cos \varphi-\bar{y} \sin \varphi \\
& y=\bar{x} \sin \varphi+\bar{y} \cos \varphi
\end{aligned}
$$

to the equation (1). We obtain

$$
\begin{aligned}
& (\bar{x} \sin \varphi+\bar{y} \cos \varphi-d)^{2}= \\
& \quad k(\bar{x} \cos \varphi-\bar{y} \sin \varphi-a)(\bar{x} \cos \varphi-\bar{y} \sin \varphi-b)(\bar{x} \cos \varphi-\bar{y} \sin \varphi-c)
\end{aligned}
$$

what is an equation with six parameters $a, b, c, d, k$ and $\varphi \in[0,2 \pi)$. 

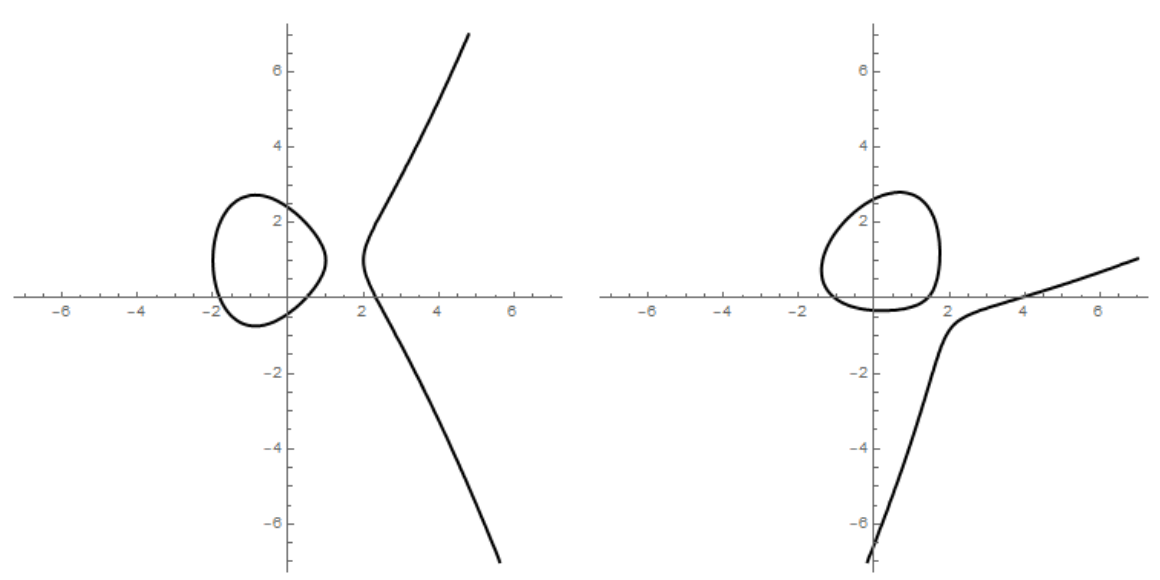

Figure 3. The elliptic curve with $a=-2, b=1, c=2, d=1, k=\frac{1}{2}$ and the same once again after the rotation with $\varphi=\frac{\pi}{4}$.

This equation can be transformed into the form

$$
\begin{aligned}
& \left(\bar{x} \sqrt{1-f^{2}}+\bar{y} f-d\right)^{2}= \\
& \quad k\left(\bar{x} f-\bar{y} \sqrt{1-f^{2}}-a\right)\left(\bar{x} f-\bar{y} \sqrt{1-f^{2}}-b\right)\left(\bar{x} f-\bar{y} \sqrt{1-f^{2}}-c\right)
\end{aligned}
$$

or

$$
\begin{aligned}
& \left(-\bar{x} \sqrt{1-f^{2}}+\bar{y} f-d\right)^{2}= \\
& \quad k\left(\bar{x} f+\bar{y} \sqrt{1-f^{2}}-a\right)\left(\bar{x} f+\bar{y} \sqrt{1-f^{2}}-b\right)\left(\bar{x} f+\bar{y} \sqrt{1-f^{2}}-c\right),
\end{aligned}
$$

where $f \in[-1,1]$.

\subsection{Curves going through points of a hexagon}

Let us present an example. We consider points $P_{1}=\left[-4, \frac{5}{2}\right], P_{2}[-3,-1]$, $P_{3}=\left[-2, \frac{7}{2}\right], P_{4}=[0,-4], P_{5}=\left[\frac{7}{2}, \frac{11}{4}\right], P_{6}=\left[5,-\frac{11}{2}\right]$. 
ON ELLIPTIC CURVES WITH A CLOSED COMPONENT PASSING

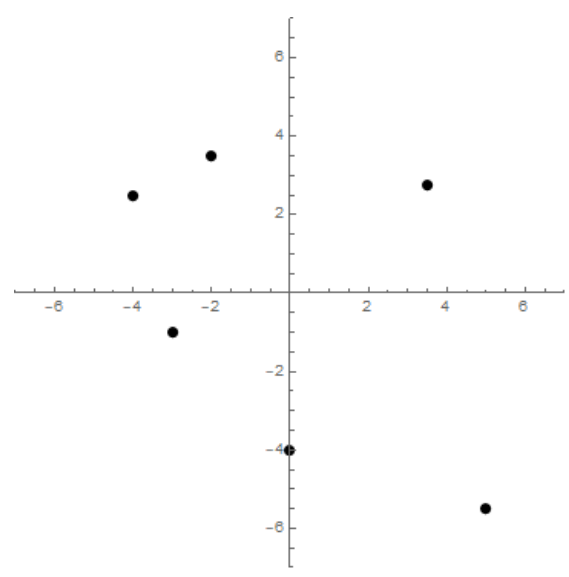

Figure 4. Points $P_{i}, i=1, \ldots, 6$.

After substituting of points, we obtain systems of non-linear equations from (3) or (4), respectively, which is impossible to solve exactly in general. So the problem must meet some approximation techniques. In our example, we have calculated the following numerical solutions:

\begin{tabular}{|c|c|c|c|c|c|c|}
\hline$a$ & $b$ & $c$ & $d$ & $k$ & $f$ & $\varphi$ \\
\hline \hline-4.05332 & 1.47818 & 3.13247 & -0.529174 & 3.18868 & -0.973143 & 2.90931 \\
\hline-3.78232 & 4.13993 & 6.84562 & 3.57652 & 0.597016 & -0.331282 & 1.90846 \\
\hline-3.76259 & 2.27353 & 3.99177 & -0.194822 & 0.385188 & 0.0619459 & 1.50881 \\
\hline-8.48145 & 4.70739 & 14.0004 & -0.288162 & 0.0209834 & -0.880181 & 3.63614 \\
\hline-3.03541 & 4.6292 & 4.73846 & -1.36848 & 0.538664 & 0.666438 & 5.44181 \\
\hline
\end{tabular}

Graphically, 
ON ELLIPTIC CURVES WITH A CLOSED COMPONENT PASSING

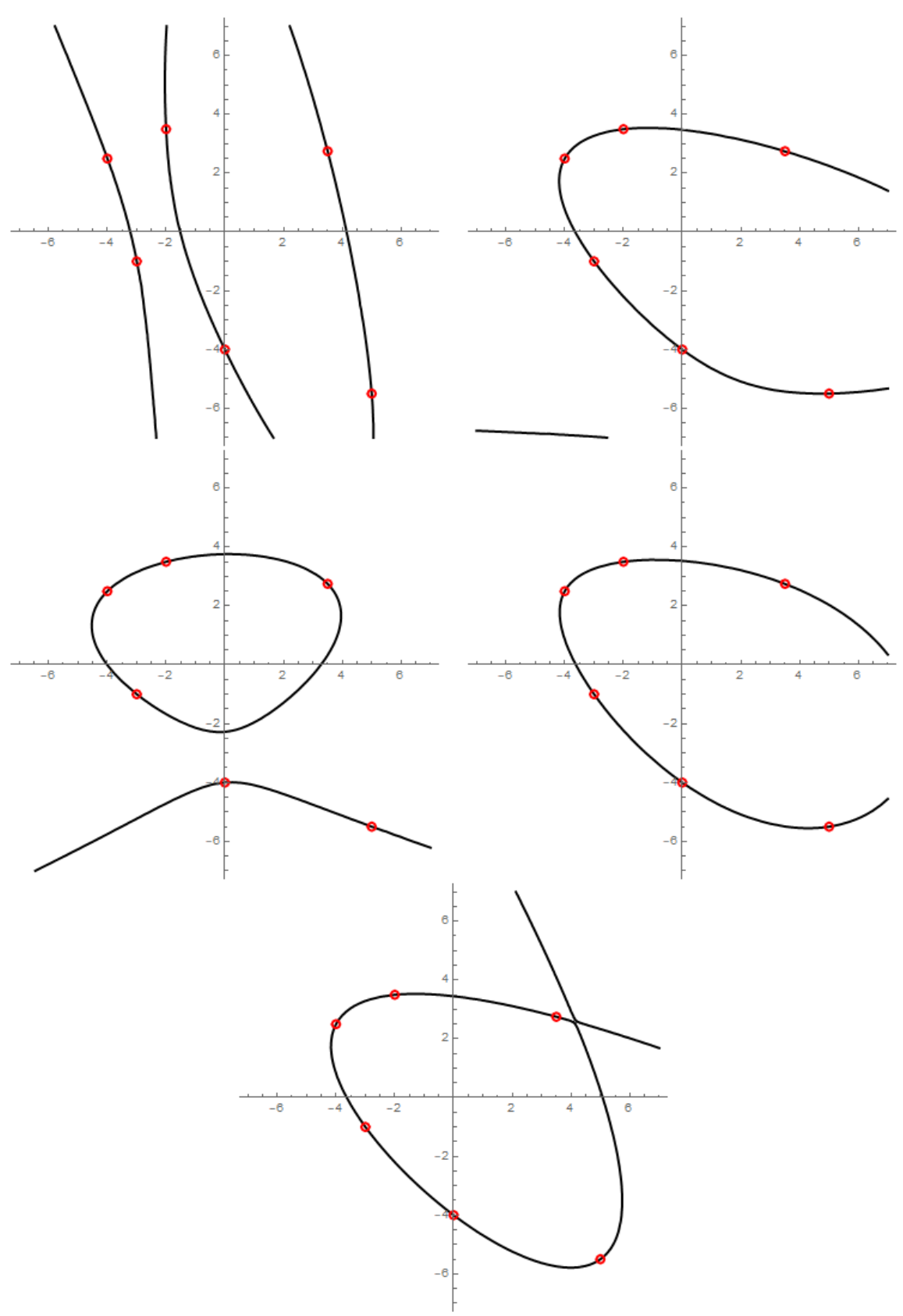

FIGURE 5. Elliptic curves of the form (3) going through the given points. 
In order to decide whether (all) points lie on the closed component of the curve or not, we apply the reverse rotation

$$
\begin{aligned}
& \bar{x}=x \cos (2 \pi-\varphi)-y \sin (2 \pi-\varphi) \\
& \bar{y}=x \sin (2 \pi-\varphi)+y \cos (2 \pi-\varphi)
\end{aligned}
$$

for the curve and the points, too. If (all) $x$-coordinates of points are less then the highest root of the cubic polynomial representing the right hand side of the elliptic curve, then the answer is positive.

Definition 1. Let us consider six points forming a convex non-degenerate hexagon. If an elliptic curve (3) is passing through these six points in such a way that $k$ points $(0 \leq k \leq 6)$ lie on a closed component, we call it the $k$-cohesive location of points on the elliptic curve.

Proposition 1. There exist convex non-degenerate hexagons possessing $n 6$ cohesive locations of points on the elliptic curve passing through their rational vertices, for $n=0,1,2,3$.

Proof. We present examples of solutions meeting the requiered condition. To save space we do not give pictures here.

(i) (no 6-cohesive location:) points $P_{1}=[-6,-4], P_{2}=\left[-6, \frac{3}{4}\right], P_{3}=$ $\left[-2, \frac{7}{2}\right], P_{3}=\left[-2, \frac{7}{2}\right], P_{4}=[0,-4], P_{5}=\left[\frac{7}{2},-\frac{15}{4}\right], P_{6}=\left[\frac{7}{2}, \frac{11}{4}\right]$.

(ii) (one 6-cohesive location:) $P_{1}=\left[-\frac{13}{2}, \frac{1}{4}\right], P_{2}=\left[-6,-\frac{1}{4}\right], P_{3}=[-2,2]$, $P_{4}=[0,-2], P_{5}=\left[\frac{7}{2}, \frac{5}{4}\right], P_{6}=[6,-1]$.

(iii) (two 6-cohesive locations:) $P_{1}=\left[-\frac{13}{2}, \frac{1}{4}\right], P_{2}=\left[-6,-\frac{1}{4}\right], P_{3}=\left[-2, \frac{27}{4}\right]$, $P_{4}=[0,-2], P_{5}=\left[\frac{7}{2}, \frac{13}{4}\right], P_{6}=[6,-1]$.

(iv) (three 6-cohesive locations:) $P_{1}=\left[-4, \frac{5}{2}\right], P_{2}=[-3,-1], P_{3}=\left[-2, \frac{7}{2}\right]$, $P_{4}=[0,-4], P_{5}=\left[\frac{7}{2}, \frac{11}{4}\right], P_{6}=\left[5,-\frac{11}{2}\right]$.

The algorithm for finding curve equations and for an eventual decision about $n$-cohesiveness using the reverse rotation has already been described.

Proposition 2. There exist convex non-degenerate hexagons possessing a $k$ cohesive location of points on the elliptic curve passing through their rational vertices, for $k=4,5,6$.

Proof. Analogously to the proof of the previous proposition, we will write an appropriate positioning of the points for particular cases when locations occur.

(i) (4-cohesive location:) points $P_{1}=\left[-4, \frac{5}{2}\right], P_{2}[-3,-1], P_{3}=\left[-2, \frac{7}{2}\right]$, $P_{4}=[0,-4], P_{5}=\left[\frac{7}{2}, \frac{11}{4}\right], P_{6}=\left[5,-\frac{11}{2}\right]$ 
(ii) (5-cohesive location:) points $P_{1}=\left[-\frac{13}{2}, \frac{1}{4}\right], P_{2}=\left[-6,-\frac{1}{4}\right], P_{3}=$ $\left[-2, \frac{27}{4}\right], P_{4}=[0,-2], P_{5}=\left[\frac{7}{2}, \frac{13}{4}\right], P_{6}=[6,-1]$.

(iii) (6-cohesive location:) points $P_{1}=\left[-4, \frac{5}{2}\right], P_{2}[-3,-1], P_{3}=\left[-2, \frac{7}{2}\right]$, $P_{4}=[0,-4], P_{5}=\left[\frac{7}{2}, \frac{11}{4}\right], P_{6}=\left[5,-\frac{11}{2}\right]$.

Remark 1. There is important in previous propositions that we are talking about rational points. ${ }^{\dagger}$ In particular, it is not sufficient to take an elliptic curve and choose six arbitrary points on it, with a suitable amount of points on its closed component and to show that these can form a non-degenerate hexagon.

Definition 2. The connected component of the elliptic curve in question will be called the spekboom curve. (This is because its shape may resemble the leaves of the plant Portulacaria afra known as spekboom in Afrikaans.)

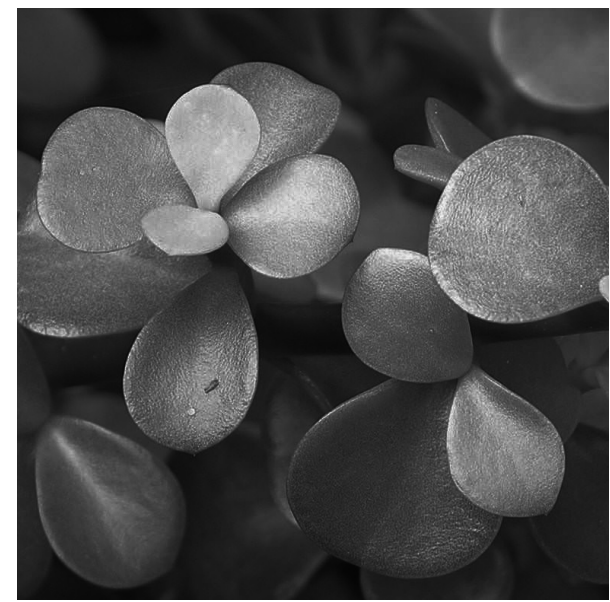

Figure 6. Leaves of Portulacaria afra (spekboom).

\section{More on hexagons}

First of all, let us note that a set of six (different) points is not determining the particular hexagon. It is essential which point follows which, so we need an

\footnotetext{
†Of course, there are connections with classical problems of an existence of rational points on a curve, for example: if $p$ is a prime number congruent to 1 modulo 4 , then there exist natural numbers $x$ and $y$ satisfying $p=x^{2}+y^{2}$ and conversely, for a prime number congruent to 1 modulo 3 there do not exist rational numbers $x$ and $y$ satisfying this equation.
} 
ordered set of six points. However, we are only concerned with non-degenerate convex hexagons, i.e. such that three vertices never lie on the same line and all inner angles are from the interval $(0, \pi)$. In that case, we do not need the ordering of the points, as it is shown in the following statement.

Proposition 3. Let $P_{i}, i=1, \ldots, 6$ are points in plane such that there is a non-degenerate convex hexagon $\mathcal{H}$ having $P_{i}$ as vertices. Then there is no non-degenerate convex hexagon having $P_{i}$ as vertices which is different from $\mathcal{H}$.

Proof. If six points in plane determine a non-degenerate convex hexagon $\mathcal{H}$, then $\mathcal{H}$ is nothing but the convex hull $P_{i}, i=1, \ldots, 6$. The convex hull is always unique.

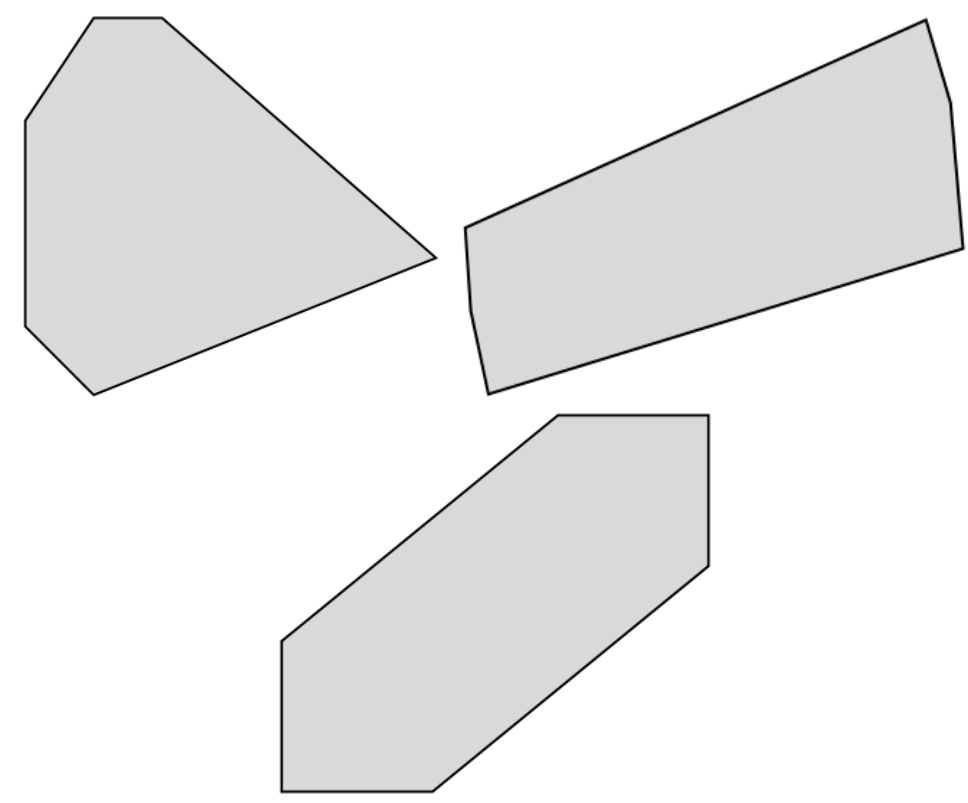

Figure 7. Examples of convex hexagons. A general hexagon, an elliptic hexagon, a right parallelo-hexagon.

\subsection{Elliptic hexagons}

By an elliptic hexagon, we mean a non-degenerate convex hexagon having all vertices on an ellipse. We remark that the property "non-degenerate convex" is, by having all points on an ellipse, already enforced. The special case is the circle hexagon having all vertices on a circle. 
Numerical experiments show that even for elliptic hexagons could occur all the cases described in Propositions 1 and 2. However, they also give rise to the following hypothesis:

Conjecture 1. There is no elliptic curve passing through the vertices of a circle hexagon.

\subsection{Right parallelo-hexagons}

By an parallelo-hexagon, we mean a hexagon with opposite sides equal and parallel (see the paper [5] of M. de Villiers). As above, we consider only convex parallelo-hexagons. By a right parallelo-hexagon, we mean a convex parallelo-hexagon with two right inner angles and such that the sides forming the right angle are of the same length.

We denote the sides forming the right angle by $u$ and the remaining two sides by $v$. Furthermore, we denote by $\gamma$ the angle as is shown on the figure. In general, we should assume that $\gamma \in\left(0, \frac{\pi}{2}\right)$, however, we just suppose that $\gamma \in\left(0, \frac{\pi}{4}\right]$ a hexagon with $\gamma \in\left(\frac{\pi}{4}, \frac{\pi}{2}\right)$ is transformable on another with $\gamma \in$ $\left(0, \frac{\pi}{4}\right)$ by one reflexion.

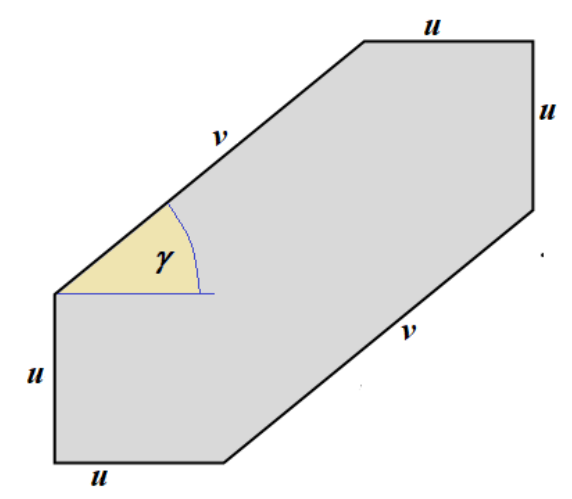

FigurE 5. The right parallelo-hexagon.

Put the left lower vertex at the origin of the coordinate system and the sides $a$ of the hexagon in alignment with the coordinate axes. Then the vertices of the right parallelo-hexagon are $[0,0],[u, 0],[u+v \cos \gamma, v \sin \gamma],[u+v \cos \gamma, u+$ $v \sin \gamma],[u \cos \gamma, u+v \sin \gamma],[0, u]$.

Proposition 4. There exist right parallelo-hexagons having infinitely many spekboom curves going through their vertices. 
Proof. It is sufficient to show an example. Let $u=1, v=\frac{1}{5}, \gamma=\frac{\pi}{4}$. Then the solution of (3), resp. (4) is one-parameter family from which one can obtain a particular solution by a specification of the parameter value: we can do so for the parameter $k$. Several values can be seen in the figure below.

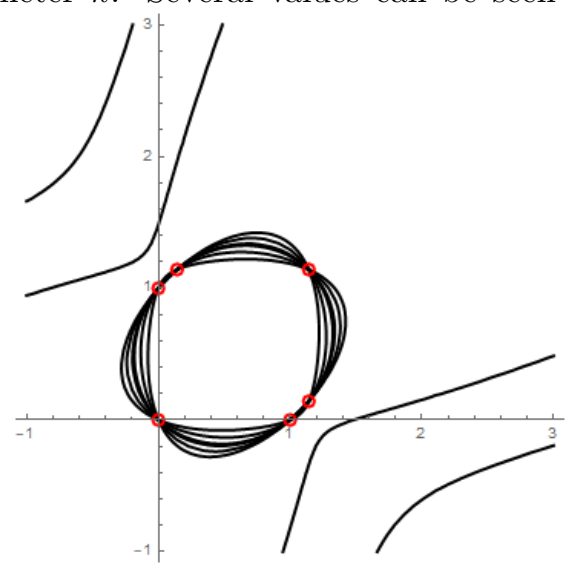

FiguRE 8. Spekboom curves of the right parallelo-hexagon with $u=1$,

$$
v=\frac{1}{5}, \gamma=\frac{\pi}{4} \text {. }
$$

\section{Properties of the spekboom curve}

Of course, there is no closed algebraic curve of the first degree. However, polygons, which are actually algebraic polynomials of the first degree "in parts", are studied extensively. Knowledge about circles ellipses as closed algebraic curves of the second degree are familiar for secondary school pupils.

However, the component of the closed curve of the third degree, which we are considering, is actually almost unnoticed until now. We write some of its elementary properties in this section. It is well-known that nonsingular elliptic curves admit no rational parametrization (by uniformization theorems), so we continue with the implicit equation. It suffices to consider a simplified equation of the form

$$
y^{2}=k x(x-a)(x-c), \quad x \leq 0, a<0<c, k>0,
$$

i.e. we take in $d=0$ and $b=0$ in (1). Moreover, we will consider the case with $k=1, a=-1$

$$
y^{2}=x(x+1)(x-c) \quad x \leq 0, c>0,
$$


as our most special case. Easy analysis leads us to the following results.

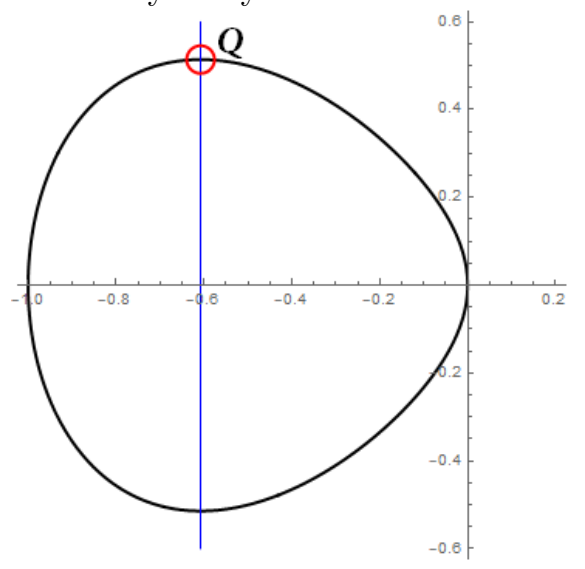

Figure 9. The spekboom curve with the marked point $Q$ in its largest width.

Proposition 5. The point of the largest width of the spekboom curve (5) is

$$
Q=\left[\frac{a+c-U}{3}, \frac{\sqrt{k(a+c-U)(2 a-c+U)(2 c-a+U)}}{3 \sqrt{3}}\right]
$$

$U=\sqrt{a^{2}-a c+c^{2}}$, in particular, for (6) it is

$$
Q=\left[\frac{c-1-\bar{U}}{3}, \frac{\sqrt{(c-1-\bar{U})(-c-2+\bar{U})(2 c+1+\bar{U})}}{3 \sqrt{3}}\right]
$$

$\bar{U}=\sqrt{1+c+c^{2}}$, and this case has limit values

$$
Q_{0}=\left[-\frac{2}{3}, \frac{2}{3 \sqrt{3}}\right] \quad\left(\text { for } c \rightarrow 0_{+}\right) \text {and } Q_{\infty}=\left[-\frac{1}{2}, \infty\right] \quad(\text { for } c \rightarrow \infty) \text {. }
$$

(If c goes to infinity, the spekboom curve becomes symmetrical and approaches the ellipse with the infinite vertical half-axis.)

Proof. Nothing but direct verification by usual differential calculus.

We finish the paper with the basic property of differential geometry of curves, which is curvature. 
Proposition 6. The curvature in the point $[-1,0]$ of the spekboom curve (6) is

$$
\kappa_{L}=\frac{2}{c+1}
$$

i.e. $\kappa_{L}=2$ for $c \rightarrow 0_{+}$and $\kappa_{L}=0$ for $c \rightarrow \infty$ and the curvature in the point $[0,0]$ of the spekboom curve (6) is

$$
\kappa_{R}=\frac{2}{c}
$$

i.e. $\kappa_{R} \rightarrow \infty$ for $c \rightarrow 0_{+}$and $\kappa_{R}=0$ for $c \rightarrow \infty$.

Proof. We compute the curvature using the formula

$$
\kappa=\frac{-\left(F_{y}^{\prime}\right)^{2} F_{x x}^{\prime \prime}+2 F_{x}^{\prime} F_{y}^{\prime} F_{x y}^{\prime \prime}-\left(F_{x}^{\prime}\right)^{2} F_{y y}^{\prime \prime}}{\left(\left(F_{x}^{\prime}\right)^{2}+\left(F_{y}^{\prime}\right)^{2}\right)^{\frac{3}{2}}}
$$

for an implicit curve $F(x, y)=0$.

Now, we can easily construct the osculating circles with the radius equal to the multiplicative inverse of the curvature.

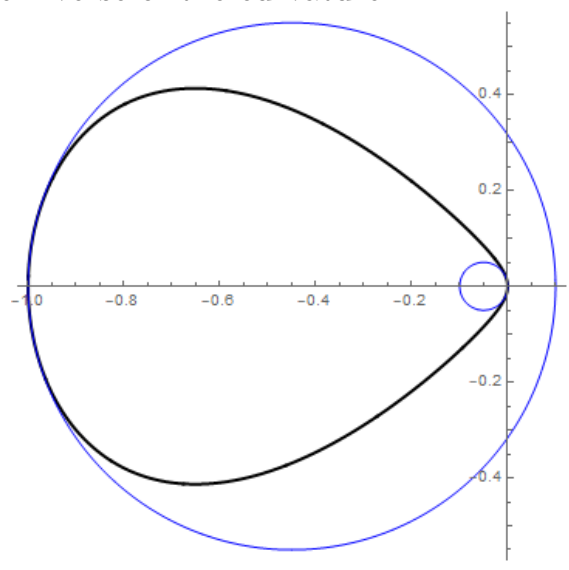

FiguRE 10. The spekboom curve (6) with $c=\frac{1}{10}$ and with osculating circles in $[-1,0]$ and $[0,0]$.

\section{References}

[1] Agarwal, M., Clifford, J. and Lachance, M., Duality and Inscribed Ellipses, Computational Methods and Function Theory 15 (4), 2015, 635644 
[2] Beltrametti, M. C., Massone, A. M. and Piana, M., Hough transform of special classes of curves, SIAM Journal on Imaging Sciences 6 (1), 2013, $391-412$

[3] Hrdina, J. and Pavlík, J., Reconstruction of an ellipse from its raster image, International Journal of Pure and Applied Mathematics 101 (2), 2015, 141-155

[4] Kopp, G. S., The arithmetic geometry of resonant Rossby wave triads, SIAM Journal on Applied Algebra and Geometry 1 (1), 2017, 352-373

[5] De Villiers, M., Some adventures in Euclidean geometry, Dynamic Mathematics Learning 2009

Acknowledgement. The author was supported by Brno University of Technology, the Specific Research Plan No. FSI-S-17-4464.

Miroslav KUREŠ,

Department of Mathematics,

Brno University of Technology,

Technická 2, 61669 Brno, Czech Republic.

Email: kures@fme.vutbr.cz 